\title{
DISKS AND DARK HALOES OF DWARF SPIRALS
}

\author{
V. FRIESE, B. FUCHS AND R. WIELEN \\ Astronomisches Rechen-Institut Heidelberg, Germany
}

We have investigated the dynamical state of the disks of a set of 9 spiral galaxies fainter than $M_{B}=-18$ and with Hubble types Scd or later for which rotation curves are available in the literature. The rotation curve of each galaxy is decomposed in disk and dark halo contributions in order to determine the mass distributions of both components. The velocity dispersion of dwarf spirals is not known, but we use the vertical scale heights of the disks assuming vertical hydrostatic equilibrium to derive estimates of the velocity dispersions. For this purpose we have performed a statistical flattening analysis of faint dwarf spirals in the ESO-Uppsala catalogue. We find that the intrinsic ratio of vertical to radial scalelengths of these types of galaxies is $z_{0} / h=0.2$ corresponding to an intrinsic flattening of $q_{0}=0.14$.

Next, we discuss the spiral structure of the galaxies in the framework of density wave theory. Toomre's stability parameter and ' $X=2$ ' criterion are evaluated for each disk. We show that the transition from Hubble type Sd to Sm or Im can be well understood from the dynamics of the disks. The transition is not correlated to the absolute brightnesses of the galaxies or the masses of the dark haloes, but to a distinct drop of the disk to dark halo mass ratios $\mathrm{D} / \mathrm{H}$ of the galaxies.

Finally, we discuss the scenario due to J. Ostriker that the dark haloes may consist of massive black holes. They would heat the galactic disks stochastically by gravitational encounters with the stars when penetrating the disks. We derive typical black hole masses of the order $10^{4}$ to $10^{5} \mathrm{M}_{\odot}$ for the low $\mathrm{D} / \mathrm{H}$ galaxies whereas we find $10^{6} \mathrm{M}_{\odot}$ for high $\mathrm{D} / \mathrm{H}$ dwarfs and giant spirals, seemingly contradicting the supposed universal nature of the black holes. But we note that the disks of the low $\mathrm{D} / \mathrm{H}$ galaxies are rather blue and have characteristically low mass-to-light ratios so that the light from these galaxies seems to be dominated by a population of rather young bright stars. Thus they might be not well suited to study the stochastic heating of galactic disks, i.e. to determine reliable black hole masses. 